\title{
Comparación entre determinaciones de gases arteriales y equilibrio acido- básico en sangre arterial y capilar "arterializada" en lactantes desnutridos severos. Evaluación del intercambio gaseoso pulmonar
}

\author{
DRES. ELIANA CERUTI *, CARLOS CASAR ${ }^{* *}$ y ARMANDO DIAZ **,
}

Las determinaciones de $\mathrm{pH}$, presión parcial de oxígeno arterial $\left(\mathrm{PO}_{2}\right)$, presión parcial de anhídrico carbónico arterial $\left(\mathrm{PCO}_{2}\right)$, exceso de base (EB) y bicarbonato standard (BS) tienen gran importancia clínica para la regulación del equilibrio ácido-básico y la evaluación de la función respiratoria. Conocidos los inconvenientes y posibles complicaciones de la extracción de sangre arterial $(1,2)$, la obtención de sangre arterializada se ha planteado con éxito como un substituto de la arterial ya desde 1922 (3). Posteriormente y especialmente en los últimos años, se han desarroIlado micrométodos que permiten las determinaciones en muestras menores incluso que $0,1 \mathrm{ml}$. $(4,5,6,7,8,9)$. La duda de si la sangre capilar realmente es representativa de la arterial ha persistido hasta ahora $\mathrm{y}$ ha sido ampliamente discutido en la literatura, tanto para el adulto como niños y recién nacidos. $(10,11$, $12,13,14,15,16,17,18)$. Diferentes autores han demostrado en el adulto y en el recién nacido normal la existencia de una buena correlación arterio-capilar entre las determinaciones de los parámetros ácido-básicos $(12,14,15,16,20,21,22$, $23,24)$ y de la presión de oxígeno arterial, especialmente cuando ésta se determina por punción del tóbulo de la oreja $(8,12,14)$. Sin embargo en un trabajo anterior (18) demostramos que tanto en los recién nacidos a término como en los prematuros sanos, sólo la determinación de los parámetros ácido-básicos en sangre capilar era representativa de los valores arteriales, no así la medición de la $\mathrm{PO}_{2}$, ya que observamos una diferencia arterio-capilar para la $\mathrm{PO}$, estadísticamente significativa en todos los niños estudiados. Estos

\footnotetext{
* Prof. Asociado de Pediatría. Departamento de Pediatria, Area Norte Escuela de Medicina U. de Chile. Jefe Laboratorio Cardiorespiratorio, Hospital Roberto del Río.

* Departamento Pediatr'a. Area Norte. Esc. Medicina U. de de Chile. Laboratorio Cardiorespiratorio, Hospital Roberto del Río.
}

resultados concuerdan por lo demás con los encontrados por otros autores $(10,17,19)$.

En los lactantes desnutridos no hay estudios al respecto. En ellos es importante evaluar el intercambio gaseoso pulmonar que no se conoce, especialmente a la luz de la demostración en ratas desnutridas de una disminución de la concentración del surfactante pulmonar (23).

Además y considerando la alta frecuencia con que presentan infecciones respiratorias graves la medición acertada de la $\mathrm{PO}_{2}$ y $\mathrm{PCO}_{2}$ permite valorar el grado de suficiencia respiratoria y por consiguiente el indicar la concentración de oxígeno adecuada y el uso de respiración asistida en los niños que la requieran. Igualmente es importante tratar racionalmente en todos ellos las frecuentes alteraciones ácido-básicas que presentan.

Por todas estas razones creemos imprescindible contar con un método sencillo que nos permita obtener muestras representativas de sangre arterial. Por eso decidimos estudiar la exacta validez de las mediciones de gases arteriales y equilibrio ácido-básico en sangre capilar, en un grupo de lactantes con đesnutrición calórico-proteica grado III y compararla con la encontrada en un grupo de lactantes con estado nutritivo normal. Fue nuestro propósito además evaluar en el primer grupo de niños, sin otra patología asociada, la función respiratoria y la regulación del equilibrio ácido-básico.

Materal y MÉToDo. En un grupo de 27 lactantes, 15 desnutridos severos sin otra patologia asociada y 12 con estado nutritivo normal, se determinaron un total de 27 pares de muestras. Las edades de todos ellos fluctuaron entre 1 y 12 meses.

Los desnutridos se catalogaron como tales considerando la relación peso/edad y peso/talla, incluyéndose en este estudio sólo aquéllos con des- 
nutrición calórico-proteica grado III con una relación peso/talla de $78 \%$ o menos $(26,27)$.

En ambos grupos se efectuaron determinaciones cn sangre arterial y capilar con un intervalo máximo entre cllas de 15 minutos.

Las muestras de sangre arterial se obtuvieron por punción directa cle la arteria femoral, en forma anaerobica, en jeringas engrasadas y heparinizadas con una concentración de $1 \mathrm{mg}$. de heparina por $\mathrm{ml}$. de sangre (28), desechándose, mediante otra jeringa, el primer ml. de sangre obtenido a través del catéter.

Las muestras de sangre arterializada se obtuvieron por punción del talon, previo calentamiento de este y de toda la extremidad inferior, a $45^{\circ} \mathrm{C}$. durante 20 minutos, siguiendo la técnica descrita por Astrup (6). La sangre se recibio en capilares heparinizados "Radiometer" que se sellaron y agitaron de acuerdo a la técnica de Andersen (5). Se tuvo especial cuidado de no presionar la extremidad del niño en el momento de obtención de la muestra.

Tanto los capilares como las jeringas se mantuyieron en hielo hasta el momento del análisis, efectuándose éste siempre dentro de los primeros 30 minutos de obtención de la sangre.

El pH se determinó en triplicado en un $\mathrm{pH}$ metro "Radiometer" 27 GM. La $\mathrm{PCO}_{2}$ se determinó directamente en un electrodo de $\mathrm{PCO}_{2}$ "Radiometer" y también indirectamente junto con la base buffer, el exceso de base y el bicarbonato standard según la técnica descrita por Astrup y Siggaard-Ardensen $(5,6)$. Como todas las mediciones de $\mathrm{pH}$ se determinaron a $38 \% \mathrm{C}$, los valores obtenidos se corrigieron a la temperatura del nino de acuerdo al factor Rosenthal: 0,0147 unidades de $\mathrm{pH}$ por grado $\mathrm{C}$ de diferencia (29). La presión parcial de oxígeno se determinó en triplicado en un electrodo de $\mathrm{O}_{2}$, se calibró con sangre equilibrada con una presión de oxígeno conocida siguiendo la técuica de Severinghaus, que consiste en caljbrar el electrodo a una presión parcial de oxígeno $5 \%$ superior a la correspondiente a la presión barométrica del momento.

En todos los niños se determinó la temperatura calórica (tele termómetro a $8 \mathrm{~cm}$. del ano), en el momento de la toma de cada muestra.

En cada muestra se determinó la concentración de hemoglobina en un espectrofotómetro "Coleman".

Resultados. Los resultados obtenidos se agruparon por separado en anbos grupos de lactantes estudiados.

En la tabla No 1 se observan las diferencias observadas entre las detcrminaciones de $\mathrm{pH}, \mathrm{PCO}_{2}$, $\mathrm{PO}_{2} \mathrm{~EB}$ y BS., en sangre arterial y arterializada en los lactantes con estado nutritivo normal. En las figuras $1^{a}, 2^{a}, 3^{a}$ y $4^{a}$ se demuestra en forma
T A B L A 1

DIFERENCIAS ENTRE LOS VALORES DE PH, PCO, $P_{2}$, EB Y BS DETERMINADOS EN SANGRE ARTERIAL Y CAPILAR EN 12 LACTANTES SANOS CON ESTADO NUTRITIVO NORMAL

\begin{tabular}{|c|c|c|c|c|c|c|c|}
\hline \multirow[b]{2}{*}{$\begin{array}{c}\text { CASO } \\
\mathrm{N}^{\circ}\end{array}$} & \multirow[b]{2}{*}{$\begin{array}{c}E D A D \\
\text { (meres) }\end{array}$} & \multirow[b]{2}{*}{$\begin{array}{c}\text { PESO } \\
\left(\mathrm{g} H^{\mathrm{H}}\right)\end{array}$} & \multicolumn{5}{|c|}{ DIFERENCIAS ARTERIO-CAPILARES } \\
\hline & & & $\Delta p H$ & $A^{P C O}$ & $\triangle P O_{2}$ & $\triangle^{E B}$ & $\triangle B S$ \\
\hline 1 & 5 & 7.260 & 0,013 & $-2,5$ & 3,1 & 0,5 & 0,4 \\
\hline 2 & 2 & 5.170 & $-0,006$ & 0,8 & 5,5 & $-0,1$ & 0,1 \\
\hline 3 & 4 & 5.300 & 0,005 & -0.7 & 1,9 & 0.7 & 0,6 \\
\hline 4 & 5 & 5.720 & $-0,018$ & 2,5 & 3,5 & 0,3 & $-0,3$ \\
\hline 5 & 1 & 4.500 & 0,010 & -1.5 & 12,0 & $-0,2$ & 0,4 \\
\hline 6 & 4 & 6,050 & $-0,003$ & 1,5 & 12,5 & 0,0 & 0,0 \\
\hline 7 & 3 & 5350 & $-0,010$ & 3,9 & 3,3 & $-0,5$ & $-0,7$ \\
\hline 8 & 2 & 4.850 & 0,014 & $-2,8$ & 9,0 & $-0, \overline{1}$ & 0.6 \\
\hline 9 & 6 & 7.575 & $-0,004$ & 1,0 & 6,5 & $-0,5$ & 1.0 \\
\hline 10 & 9 & 9.3000 & 0,015 & $-1,8$ & 6.0 & 0,9 & 0,9 \\
\hline 11 & 8 & 8.950 & 0,019 & $-2,0$ & 6,4 & 0,7 & $-0,9$ \\
\hline 12 & 10 & 9.480 & $\rightarrow 0,014$ & $-2,0$ & 8,1 & $-0,2$ & $-0,7$ \\
\hline
\end{tabular}

pH: unidades

$\mathrm{PCO}_{2}:$ mrithg.

$\mathrm{PO}_{2}:$ mmHy.

$\mathrm{EB}^{2}: \mathrm{mEq} / \mathrm{L} / \mathrm{sangre}$

ES: $m E q / L /$ plasma.

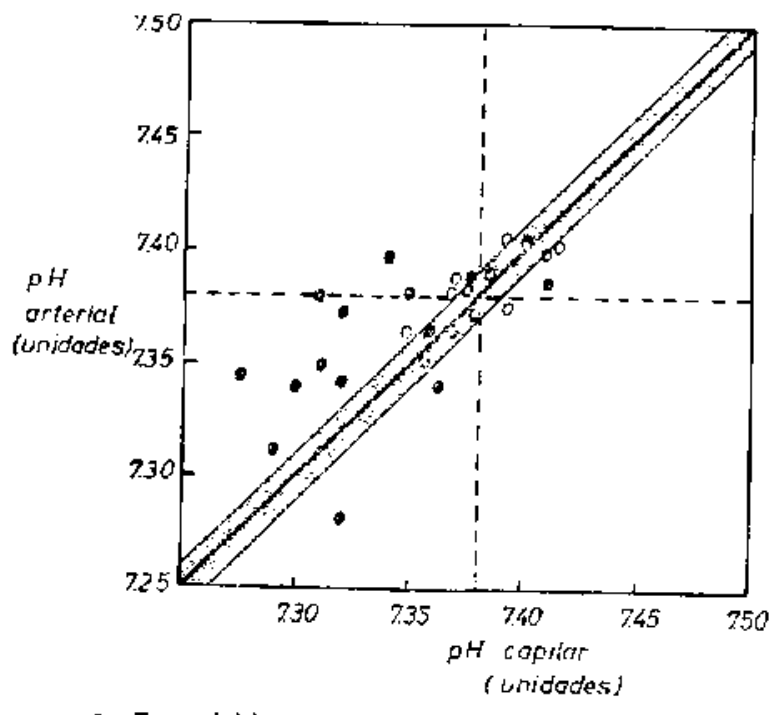

- Desnutridos severos.

- Estado nutritivo normol.

Figura 1.- Comparación entre las determinaciones de $\mathrm{pH}$ en sangre arterial $y$ arterializada (capilar) en lactantes desnutridos severos 9 luctantes con estadó nutritivo normal. (El área sombreada representa el rango de error del método, o.,ol unidades).

gcáfica la correlación observada entre ambos métodos para el pH, $\mathrm{PCO}_{2}, \mathrm{PO}_{2}$ y E. B. En la tabla No 2 se muestran las diferencias arterio-capilares promedio \pm E.S. para los mismos parámetros en lactantes normales observándose una excelente correlación estadística entre ambos métodos excepto en lo que se refjere a la determinación de 


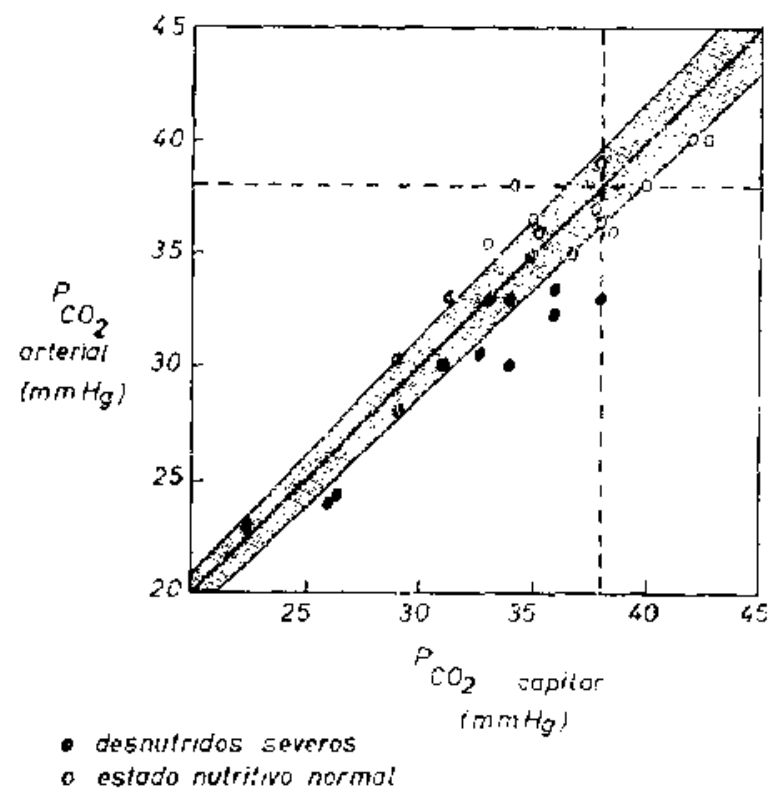

Figura 2.- Comparación entre las determinaciones de $\mathrm{PCO}$ en Figura 2.- Comparacion entre las detercante en lactantes desnusangre arterjal y "Artetializada" (capilar) en lactantes dcsnutridos severos y lactantes con estado ritritivo normal. (El area
sombreada representa el tango de crror del ntétodo, 5\%).

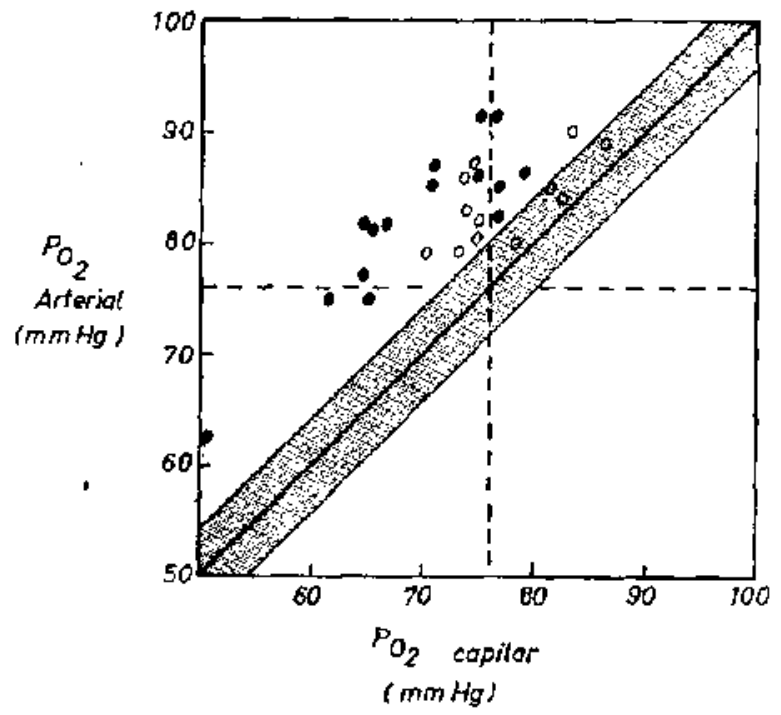

- Desnutridos severos.

- Estodo nutritivo normal.

Fina $3-$ Comparacion entre las determinaciones de $\mathrm{PO}$ en Figura 3.- Comparacializada (capilar) en laclantes desnutridos sangre arterial y arterial estado nutritivo normal. (Bl área sombreada representa el rango de error del método, $3 \mathrm{~mm} \mathbf{\mathrm { gg }}$.).

la $\mathrm{PO}_{2}$. Se demuestra que el promedio de las diferencias entre las determinaciones de $\mathrm{pH}$ en sangre arterial y capilar fue de $0,0017 \pm$ ES 0,003 unidades $(\mathrm{p}<0,25)$ con un rango que varía entre 0.018 y 0,019 unidades. El promedio de la diferencia para la $\mathrm{PCO}_{2}$ fue de $0,3 \pm$ ES 0,62 $\mathrm{mmHg}(\mathrm{p} .<0,30)$ con un rango fluctuante entre $-2,8$ y $3,9 \mathrm{mmHg}$.

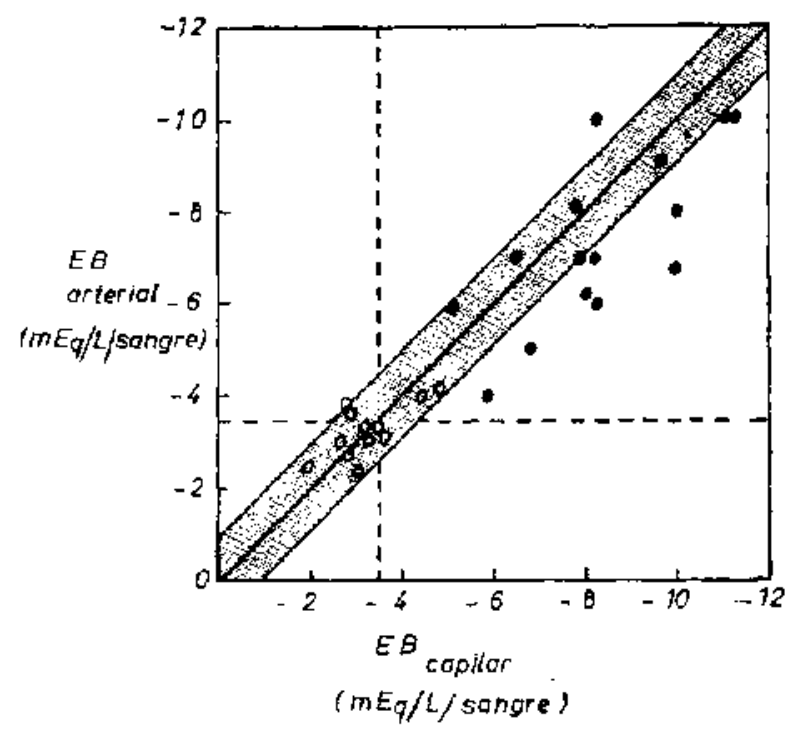

- Desnutridos.

- Estoco nutritivo normal.

Figura 4.- Comparación entre las determinaciones de Exceso de Base (EB) cn sangre arterial $y$ arterializada (capilar) en lactantes desnutridos severos y lactantes con estado nutritivo lactantes desnutridos suces representa el rango de error del método nats o menos $1 \mathrm{mEq} / \mathrm{L}$ ).

El estudio del componente metabólico del equilibrio ácido-base en lactantes bien nutridos muestra resultados similares. En ninguno de ellos se observaron diferencias significativas entre las determinaciones arterio-capilares de EB y ES.

De los resultados expuestos en la tabla No 2 se desprende que en los lactantes con estado nutritivo normal las diferencias entre las determinaciones arterio-capilares para los parámetros del equilibrio ácido-básico no son significativas. En ninguno de los niños cstudiados se observaron diferencias entre las determinaciones de $\mathrm{pH}$ mayores de 0.020 unidades $y$ entre las determinaciones de $\mathbf{P C O}_{2}$ mayores de $3,9 \mathrm{mmHg}$.

En este mismo grupo de niños se demostró que la diferencia arterio-capilar para la $\mathbf{P O}_{2}$ fue siempre significativa, variando entre 1,9 y $12,5 \mathrm{mmHg}$. con un promedio de $6,5 \mathrm{mmHg}$. $\pm \mathrm{ES}, 1,35$ (p. $<0,0001$ ) (tabla No 2 y Fig. No 3 ). Sin embargo y como se observará más adelante, las diferencias observadas en los lactantes bien nutridos son significativamente menores que las encontradas en los desnutridos severos.

En el grupo de lactantes con desnutrición ca1órico-proteico grado III las diferencias arteriocapilares encontradas para todos los parámetros fueron estadísticamente significativas y mayores que las observadas en los niños bien nutridos (Tablas № 3 y 4, Fig. 1, 2, 3, y 4). Las diferencias arteriocapilares para el $\mathrm{PH}$ variaron entre $-0,040$ y 0,070 unidades con una diferencia promedio de $0,022 \pm$ ES $0,008(p<0,005)$. En 
T A B L A 2

PROMEDIO \pm ES DE LAS DIFERENCIAS ENTRE LAS DETERMINACIONES DE pH, PCO, PO, EB Y ES EN SANGRE ARTERIAL Y ARTERIALIZADA EN 12 LACTANTES SANOS CON ESTADO NUTRITIVO NORMAL

\begin{tabular}{l|ccccc}
\hline & $\mathrm{pH}$ & $\mathrm{PCO}_{2}$ & $P O_{2}$ & $E S$ & $B S$ \\
\hline Promedio diferencias & 0,0017 & $-0,3$ & 6,5 & $-0,04$ & $\mathbf{0 , 1 6}$ \\
Rango & $-0,018 \mathrm{a}$ & $-2,8 \mathrm{a}$ & $1,9 \mathrm{a}$ & $-0,7 \mathrm{a}$ & $-\mathbf{0 , 9} \mathbf{a}$ \\
& $\mathbf{0 , 0 1 9}$ & 3,9 & 12,5 & $\mathbf{0 , 9}$ & $\mathbf{1 , 0}$ \\
D. S. promedio diferencias & 0,012 & 2,17 & $\mathbf{4 , 6 6}$ & $\mathbf{0 , 5 3}$ & $\mathbf{0 , 6 4}$ \\
E. S. promedio diferencias & $\mathbf{0 , 0 0 3}$ & 0,62 & 1,35 & $\mathbf{0 , 1 5}$ & $\mathbf{0 , 1 8}$ \\
$\mathrm{t}$ & $\mathbf{0 , 5 6}$ & 0,48 & $\mathbf{4 , 8 1}$ & $\mathbf{0 , 2 6}$ & $\mathbf{0 , 8 7}$ \\
$\mathbf{P}$ & $<\mathbf{0 , 2 5}$ & $<0,30$ & $<0,0001$ & $<\mathbf{0 , 3 5}$ & $<0,20$ \\
\hline
\end{tabular}

pH: unidades.

$\mathrm{PCO}_{2}$ : mmHg.

EB: $\mathrm{mEq} / \mathrm{L} / \mathrm{sangre}$

$\mathrm{PO}_{2}$ : $\mathrm{mmHg}$.

$\mathrm{ES}: \mathrm{mEq} / \mathrm{L} / \mathrm{plasma}$.

TA B L A ?

DIFERENCLAS ENTRE LOS VALORES DE PH, PCO, PO RIAL Y CAPILAR EN IS LACTANTES CON DESNUTRICION CALORICA-PROTEICA GRADO WI.

\begin{tabular}{|c|c|c|c|c|c|c|c|c|}
\hline $\begin{array}{c}\text { Caso } \\
N^{o}\end{array}$ & $\begin{array}{r}\text { Edad } \\
(m s)\end{array}$ & $\begin{array}{c}\text { Relacion } \\
\text { Peso/edad } \\
\%\end{array}$ & $\begin{array}{c}\text { Relación } \\
\text { Peso/talla } \\
\%\end{array}$ & $\triangle p H$ & $\begin{array}{l}\text { Diferencias } \\
\triangle \mathrm{PCO}_{2}\end{array}$ & $\begin{array}{c}\text { Arterio - } \\
\triangle \mathrm{PO}_{2}\end{array}$ & $\begin{array}{l}\text { silares } \\
\qquad \triangle E B\end{array}$ & $\Delta \boldsymbol{B S}$ \\
\hline $\mathbf{I}$ & 3 & 48 & 72 & 0,070 & $-5,0$ & 12,0 & $-2,0$ & 1,3 \\
\hline 2 & 9 & 44 & 65 & 0,040 & $-2,5$ & 8,4 & $-1,8$ & 1,3 \\
\hline 3 & 4 & 47 & $<70$ & 0,070 & $-5,0$ & 15,7 & $-1,8$ & 1,7 \\
\hline 4 & 3 & 65 & 72 & $-0,025$ & 0,4 & 14,0 & $-1,0$ & 0,9 \\
\hline 5 & 5 & 67 & 78 & 0,021 & $-1,0$ & 7,3 & $-1,0$ & 0,6 \\
\hline 6 & 11 & 66 & 68 & 0,022 & $-1,0$ & 15,8 & $-0,8$ & 0,5 \\
\hline 7 & 4 & 62 & 75 & 0,053 & $-1,0$ & 12,5 & $-2,1$ & 1,7 \\
\hline 8 & 5 & 52 & 75 & 0,005 & $-2,0$ & 15,6 & 0,3 & 0,0 \\
\hline 9 & 8 & 47 & 63 & $-0,040$ & 0,0 & 13,5 & 1,8 & $-1,3$ \\
\hline 10 & 5 & 54 & 78 & 0,040 & 0,0 & 16,0 & $-2,2$ & 1,6 \\
\hline 11 & 3 & 62 & 77 & 0,007 & 1,3 & 14,5 & $-1,0$ & 0,6 \\
\hline 12 & 4 & 59 & 72 & 0,012 & $-2,2$ & 8,3 & 0,8 & $-0,5$ \\
\hline 13 & 5 & 55 & 62 & 0,031 & $-2,0$ & 5,5 & $-0,5$ & 0,3 \\
\hline 14 & 4 & 60 & 68 & 0,059 & $-3,7$ & 12,0 & $-1,9$ & 1,5 \\
\hline 15 & 5 & 61 & 72 & $-0,022$ & 1.7 & 16,0 & 0,5 & $-0,5$ \\
\hline
\end{tabular}

pH: unidades

$\mathrm{PCO}_{2}$ : $\mathrm{mmHg}$

$\mathrm{PO}_{2}: \mathrm{mmHg}$
ES: $\mathrm{mEq} / \mathrm{L} /$ sangre

BS: $\mathrm{mEg} / \mathrm{L} / \mathrm{plasma}$
12 de los 15 niños desnutridos severos las diferencias para el pH fueron mayores de 0,020 unidades, no observándose esta magnitud de diferencias en ninguno de los lactantes normales estudiados.

En los desnutridos las diferencias observadas para la $\mathrm{PCO}_{2}$ tambiến fueron mayores que las encontradas en los normales variando entre $-5,0$ a $1,7 \mathrm{mmHg}$. con una diferencia arterio-capilar pro- medio de $-1,46 \pm$ ES $0,52 \mathrm{mmHg}(\mathrm{p}<0,005)$.

En los lactantes desnutridos severos las diferentias arteriocapilares promedio para el EB y el BS fueron también significativas, aun cuando el rango de variación fue muy estrecho. La diferencia promedio para el ES fue de $-0,84 \pm$ ES 0,31 ( $\mathrm{p}<0,005$ ) con un rango entre $-2,2$ y $1,8 \mathrm{mEq} /$ $\mathrm{L} /$ sangre. La diferencia promedio para el BS fue 
T A B L A 4

PROMEDIO \pm ES DE LAS DIFERENCIAS ENTRE LAS DETERMINACIONES DE $p H, P_{2} \mathrm{O}_{2}, \mathrm{PO}_{2}$, EB Y BS EN SANGRE ARTERIAL Y ARTERIALIZADA EN 15 LACTANTES CON DESNUTRICION

\begin{tabular}{l|ccccc}
\hline & $p H$ & $P C O$ & $P O_{2}$ & $E B$ & $B S$ \\
\hline Promedio diferencias & 0,022 & $-1,46$ & 12,5 & $-0,84$ & 0,65 \\
Rango & $-0,040$ a & $-5,0$ a & 5,5 a & $-2,2$ a & $-1,3$ a \\
& 0,034 & 1,7 & 16,0 & 1,8 & 1,7 \\
DS. promedio diferencias & 0,034 & 2,04 & 3,51 & 1,21 & 0,92 \\
ES. promedio diferencias & 0,008 & 0,52 & 0,90 & 0,31 & 0,23 \\
t & 2,75 & 2,77 & 13,8 & 2,70 & 2,82 \\
P & $<0,005$ & $<0,005$ & $<0,0005$ & $<0,005$ & $<0,005$ \\
\hline
\end{tabular}

$\mathrm{pH}$ : Unidades

$\mathrm{PCO}_{2}$ : $\mathrm{mmHg}$.

$\mathrm{PO}_{2}$ : $\mathrm{mmHg}$

de $0,65 \pm$ ES 0,23 ( $p<0,005)$ con un rango entre $-1,3$ y $1,7 \mathrm{mEq} / \mathrm{L} /$ plasma.

En el grupo de lactantes desnutridos severos las diferencias entre la $\mathrm{PO}_{2}$ determinada en sangre arterial y arterializada fueron siempre significativas $\mathrm{y}$ francamente mayores que las observadas en niños normales ( tabla Nơ 4 y Fig. 3 ). El promedio de las diferencias para la $\mathrm{PO}_{2}$ fue de $12,5 \mathrm{mmHg}$. \pm ES $0,90 \mathrm{mmHg}$. ( $p<0,0005$ ) y el rango varió entre 5,5 y $16 \mathrm{mmHg}$.

Finalmente, en la tabla No 5 se observan los valores arteriales promedio $\pm \mathrm{DS}$ de $\mathrm{pH}, \mathrm{PCO}_{3}$, $\mathrm{PO}_{2}$, EB y BS en ambos grupos de lactantes estudiados, demostrándose que en ambos la $\mathrm{PO}_{2}$ fue totalmente normal. Solamente en un lactante desnutrido se encontró una $\mathrm{PO}_{2}$ inferior al límite considerado normal (Fig. 3). Posteriormente se comprobó en este enfermo la presencia de un ductus hipertenso con inversión del shunt. Se observó además en los lactantes desnutridos la presencia de una acidosis metabólica moderada parcialmente compensada por hiperventilación (X pH 7.360, x $\mathrm{PaCO}_{2} 30,04 \mathrm{mmHg}, x \mathrm{~EB}-7,38 \mathrm{mEg} / \mathrm{L} / \mathrm{san}-$ gre y X BS $18,3 \mathrm{mEq} / \mathrm{L} /$ plasma). Al analizar los resultados individualmente se encontró que en 7 de ellos la acidosis metabólica estaba parcialmente compensada y que en los restantes 8 estaba totalmente compensada por hiperventilación.

Discusión. Siendo el propósito fundamental de los procesos dinámicos de la respiración la mantención de presiones parciales de oxígeno y anhídrido carbónico normales en sangre arterial, el conocimiento de esas presiones permite evaluar el grado de suficiencia de la respitación. Es importante destacar que la determinación de la $\mathrm{PO}_{2}$ y $\mathrm{PCO}_{2}$ en sangre venosa no sirve para los fines anteriores ya que en la sangre venosa la concentración de oxígeno y anhídrico carbónico depende
EB: $m E q / L /$ sangre

ES: $\mathrm{mEq} / \mathrm{L} /$ plasma.
TA B L A 5

VALORES PROMEDIO \pm DS DE PH, $\mathrm{PCO}_{2}, \mathrm{PO}_{2}, \mathrm{~EB}$ $Y$ BS EN SANGRE ARTERIAL EN 12 LACTANTES CON ESTADO NUTRITIVO NORMAL Y IS LACTANTES CON DESNUTRICION CALORICO. PROTEICA GRADO III

Lactantes con estado nutritivo normal:

\begin{tabular}{|c|c|c|c|c|}
\hline & $\overline{\boldsymbol{X}}$ & \pm & $D S$ & \\
\hline pH (unidades) & 7.382 & & \pm & 0,017 \\
\hline $\mathrm{PCO}_{2}(\mathrm{mmHg})$ & 37,25 & & \pm & 1,76 \\
\hline $\mathrm{PO}_{2}(\mathrm{mmHg})$ & 83,75 & & \pm & 3,76 \\
\hline $\mathrm{EB}(\mathrm{mEq} / \mathrm{L} /$ sangre $)$ & $-3,2$ & & \pm & 0,56 \\
\hline BS $(\mathrm{mEq} / \mathrm{L} / \mathrm{plasma})$ & 19,85 & & \pm & 0,70 \\
\hline
\end{tabular}

Lactantes con desnutrición calorico proteica grado $I I I$.

$$
\overleftrightarrow{\boldsymbol{X}} \quad \pm \quad D S
$$

\begin{tabular}{l|ccl}
\hline pH (unidades) & 7,360 & \pm & $\mathbf{0 , 0 3 4}$ \\
$\mathrm{PCO}_{2}$ (mmHg) & 30,04 & \pm & 3,78 \\
$\mathrm{PO}_{2}(\mathrm{mmHg})$ & 81,20 & \pm & 7,9 \\
$\mathrm{~EB}(\mathrm{mEg} / \mathrm{L} /$ sangre) & $-7,38$ & \pm & $\mathbf{1 , 8 6}$ \\
$\mathrm{BS}(\mathrm{mEq} / \mathrm{L} /$ plasma) & 18.31 & \pm & $\mathbf{1 , 4 0}$ \\
\hline
\end{tabular}

no sólo de la concentración de esos gases en la sangre arterial sino también, y en forma muy importante, del metabolismo celular y del flujo sanguíneo tisular. Considerando además, que la san- 
gre arterial es la base de referencia más importante para el estudio ácido-básico, es lógico contar con algún método representativo y sencillo que permita evaluar los gases arteriales y el equilibrio ácido-básico en los lactantes, muy especialmente en los desnutridos severos tan afectos a presentar complicaciones respiratorias graves y trastornos profundos del equilibrio áciđo-básico e bidroelectrolítico.

Este estudio demuestra que on el lactante sano con buen estado nutritivo existe una excelente correlación arterio-capilar para el $\mathrm{pH}, \mathrm{PCO}_{2}, \mathrm{~EB}$ y B.S. Estos resultados concuerdan con, los obtenidos por algunos autores especialmente, en adultos $(11,12,14,16,20,21,22,23,24)$ y por lo observado por nosotros $y$ otros investigadores en recién nacidos a término y prematuros sanos $(10$, $15,18,19)$. Al igual que en nuestra observación anterior en recién nacidos normales y prematuros sanos, volvimos a demostrar en el lactante normal que no existe una correlación arterio-capilar satisfactoria para la $\mathrm{PO}_{2}$. Teniendo presente este último hecho podemos concluir que en el Iactante sano con buen estado nutritivo, la sangre capilar que fluye libremente bajo circunstancias normales es virtualmente arteriolar y por ende, las determinaciones de los diversos parámetros del equilibrio ácido-básico en sangre capilar son representativas de las observadas en sangre arterial. También en los lactantes bjen nutridos observamos una excelente correlación en presencia de hiperventilación hecho que corrobora los hallazgos de Knudsen en adultos (23) y también durante el ejercicio lo que igualmente está de acuerdo con algunas observaciones realizadas en adultos $(11,21)$.

En los lactantes desnutridos severos las diferencias arterio-capilares para el $\mathrm{pH}, \mathrm{PCO}_{2}, \mathrm{~EB}$ y ES fueron siempre significativas, al contrario de lo observado èn lactantes sanos. En los desnutridos, la sangre capilar mostró un $\mathrm{pH}$ más bajo, una $\mathrm{PCO}_{2}$ más elevada y una menor $\mathrm{PO}_{2}$ que la sangrearterial. Estas diferencias observadas en los desnutridos calóricos proteicos grado ШI podrían explicarse por la presencia de una vasoconstricción de los capilares cutáneos con la consiguiente disminución de la velocidad de circulación capilar, es decir, por la existencia de una perfusión tisular disminuída que se manifiesta en estos enfermos por palidez, enfriamiento y cianosis. Ya hace muchos años atrás se había comprobado en los desnutridos un espasmo de los capilares cutáneos y al examen microscópico una disminución significativa de la velocidad circulatoria a nivel capilar $(30,31,32)$. Estas alteraciones bemodinámicas presentes en los desnutridos severos hacen no significativos los resultados obtenidos en sangre capilar, especialmente en lo que se refiere a la $\mathrm{PO}_{2}$, y por cierto, no conparables a los observados en lactantes normales con una adecuada circulación periférica.

Es posible además, que en el desnutrido severo con una pobre perfusión periférica, no se pueda obtener una adecuada vasodilatación aun con una têcnica de calentamiento satisfactoria, ya que cs síbido que en estos enfermos, la vasodilatación locitizada como respuesta al calentarmiento no ocure con la misma rapidez y frecuencia que en el lactante normal. También es probable que en el desnutrido, tal como se ha demostrado en el recién nacido de bajo peso (18), la temperatura corporal profunda tenga marcada influencia en las diferencias encontradas entre la sangre arterial $y$ capilar. El que en este trabajo hayamos observado Ias mayores diferencias arterio-capilares en aquellos lactantes desnutridos con temperaturas corporales profundas más bajas apoya esta última hipótesis. Nuestros resultados permiten destacar la importancia del calentamiento mantenido y prolongado como un medio útil de reducir los márgenes de diferencia entre la sangre arterial y capilar, especialmente cuando se utiliza el talón como sitio de punción.

Sin embargo, aun cuando todas las consideraciones anteriores deben do tenerse presente, al analizar en el grupo de lactantes desnutridos severos los resiltados individualmente, se puede observar que el rango de variación de las diferencias, si bien es significativo desde el punto de vista estadístico, no es significativo desde el punto de vista clínico, a menos que se esté en presencia de una acidosis muy severa (en estas circustancias, las muestras siguientes deberán obtenerse de una arteria y no a través de una punción capilar). Salvo esta última eventualidad creemos que desde eI punto de vista de su aplicación clínica práctica, los valores de $\mathrm{pH}, \mathrm{PCO}_{2}, \mathrm{~EB}$ y $\mathrm{BS}$ determinados en sangre capilar pueden ser utilizados con confiabilidad en la evaluación de las alteraciones ácidobásicas del desnutrido severo. Asimismo, los resultados obtenidos hacen posible que la determinación de la $\mathrm{PCO}_{2}$ en sangre capilar pueda ser considerada como un índice de ventilación alveolar.

En el presente estudio volvimos a demostrar, lo mismo que habíamos comprobado en los recién nacidos a término y prematuros sanos (18), es decir que la determinación de la $\mathrm{PO}_{2}$ en sangre capilar no es representativa de la $\mathrm{PO}_{2}$ arterial ni en los lactantes sanos ni mucho menos en los desnutridos severos. El que Ia diferencia arterio-capilar para la $\mathrm{PO}_{2}$ sea siempre significativa no es sorprendente ya que varios autores habian observado en adultos una deficiente correlación arteriocapilar para la $\mathrm{PO}_{2}$ cuando ésta se determinaba en muestras obtenidas por punción del dedo en vez del lóbulo de la oreja (8).

Las mayores diferencias arterio-capilares ob- 
servadas para la $\mathrm{PO}_{2}$ que para la $\mathrm{PCO}_{2}$ y el $\mathrm{pH}$ pueden explicarse si se considera que en los capilares siempre se produce una pequeña mezcla con sangre venosa, aun cuando el calentamiento sea adecuado. Esta pequeña mezcla no alcanza a afectar significativamente ni a la $\mathrm{PCO}_{2}$ ni al $\mathrm{pH}$, por lo menos en los lactantes normales, pero sí afecta el \% de saturación de la hemoglobina. Así, una pequeña caída de este porcentaje determina una disminución significativa de la $\mathrm{PO}_{2}$ como consecuencia de la forma de la curva de disociación de la hemoglobina (17).

La deficiente correlación observada entre la $\mathrm{PO}_{2}$ arterial y capilar tiene especial significado clínico en los lactantes desnutridos si se considera que éstos presentan con gran frecuencia afecciones respiratorias graves que los llevan a la insuficiencia respiratoria, requiriendo, por lo tanto, de la administración de una mayor concentración de oxígeno en el aire inspirado. En estas circunstancias, cuando da $\mathrm{PaO}_{2}$ sube sobre los $100 \mathrm{mmHg}$, las diferencias anterio-capilares son considerablemente mayores (17) hecho que le resta aún mayor validez a la determinación de la $\mathrm{PO}_{2}$ en sangre capilar.

Es importante destacar que en todos los desnutridos severos menos uno se observaron $\mathbf{P O}_{2}$ normales (ausencia de hipoxemia) y $\mathrm{PCO}_{2}$ normales o bajas lo que evidencia un adecuado intercambio gaseoso pulmonar. La única referencia existente en la literatura, en relación a que la concentración del surfactante pulmonar disminuiría en ratas con desnutrición no ha sido demostrada en el ser humano y requicre de un mayor estudio que abre interesantes posibilidades de investigación. Creemos que esta experiencia demuestra que el desnutrido sereno no presenta alteraciones groseras del intercambio gaseoso pulmonar aunque obliga a un estudio más profundo, especialmente de la mecánica respiratoria.

En todos los desnutridos severosı se encontró además una acidosis metabólica parcial o totalmente compensada por hiperventilación lo que también demuestra la existencia de una adecuada compesacion respiratoria. La existencia en el desnutrido, sin patología asociada, de una acidosis metabólica ya ha sido demostrada por otros autores $(33,34,35)$ pero hasta ahora no ha podido ser claramente explicada. En una próxima conuunicación comentaremos con más detalle las alteraciones ácido-básicas del desnutrido severo y algunas de sus posibles causas.

En resumen, podemos concluir que en los lactantes, cualquiera sea su estado nutritivo, puede y debe usarse sangre capilar como substituto de sangre arterial cuando se requiere evaluar el equilibrio ácido-básico, e incluso, la ventilación alveolas a través de la determinación de la $\mathrm{PCO}_{2}$. En los desnutridos, o en cualquier lactante con cir- culación venosa disminuída o con ectasis venosa marcado los parámetros respiratorios deben evaluarse en sangre arterial. Durante la exígeno-terapia, la determinación de la $\mathrm{PO}_{2}$ debe efectuarse siempre en sangre arterial.

\section{RESUMEN}

1.- En the total de 27 lactantes, 15 desmutridos severos, catalogados como tales según su relación peso/edad y peso/talla, y $I 2$ con estado nutritivo normal, se determinaron $p H$, $\mathrm{PCO}_{2}, \mathrm{PO}_{2}, \mathrm{~EB}$ y $\mathrm{BS}$ en 27 pares de muestras de sangre arterial y capilar (arterializada) $o b-$ tenidas con un intervalo de 10 a 15 minutos.

2.- En los lactantes sanos con estado nutritivo normal, el pH, la PCOz, el EB y el BS determinados en sangre capilar reflejaron fielmente los valores arteriales. Esta correlación se mantuvo en presencia de hiperventilación $y$ ejercicio.

3.- En los lactantes desnutridos severos no se observó correlación estadisticamente significativa para ninguno de los parámetros estudiados; sin embargo, al analizar las diferencias desde el punto de vista clínico, no se consideraron significativas, excepto en to que se refiere a la $\mathrm{PO}_{2}$.

4.- En ambos grupos de niños y a todas las edades, las diferencias entre las determinaciones de $\mathrm{PO}_{2}$ en sangre arterial y capilar fueron siempre significativas, lo que demuestra que la determinación de la $\mathrm{PO}_{2}$ en sangre capilar no es representativa de la efectuada en sangre arterial.

5.- En todos los desnutridos severos se observó la presencia de una acidosis metabólica parcial a totalmente compensada por hiperventilación.

6.- Se disculen las hipótesis que explicarian las diferencias encontradas entre los lactantes con estado nutritivo normal y los desnutridos severos.

7.- En resumen, se concluye que en el lactante, cualquiera sea su estado nutritivo, puede $y$ debe utilizarse sangre capilar para las determinaciones ácido-básicas, incluso para evaluar lo ventilación alveolar. En el desnutrido o en cualquier lactante con circulación capilar disminuida o con ectasis venoso, los parametros respiratorios deben evaluarse en sangre arterial. 


\section{SUMMARY}

1.-A total of 27 pairs of arterial and capillary sam. ples were obtained in 27 infants ( 1 to 12 months); of these 12 were healthy children and the remaining 15 had severe malnutrition ( $30 \%$ or more deficit for expected weight \& heigth).

2.- In the healthy infants there was agreement between arterial and arterialized capillary blood $\mathrm{pH}, \mathrm{PCO}_{2}$, $\mathrm{BE}$ ans $\mathrm{SB}$. The agreement was maintained during hiperventilation and exercise.

3,--In the infants with severe malnutrition the differences between arterial and capillary blood $\mathrm{pH}$, $\mathrm{PCO}_{2}, \mathrm{PO}_{2}, \mathrm{BE}$ and $\mathrm{SB}$ were always significant, and greater than that observed in healthy infants.

4.- The differences observed in infants with severe malnutrition between arterial and capillary blood $\mathrm{pH}$, $\mathrm{PCO}_{2}, \mathrm{BE}$ and SB were not considered great enough to prevent cautious clinical acceptance of capillasy readings.

S. - In both groups of infants at any age, the differences between arterial and capillary blood $\mathrm{PO}_{2}$ were significant.

6.- Ali the infants with severe malnutrition had metabolic acidosis with complete or parcial respiratory compensation.

\section{Bibliografía}

1.-Ray, $S$. Complications of arterial puncture (letter to ed). Brit. Med. J. 11: 758, 1964.

2.-Mortensen, J. D. Clinical sequelae from arterial needle puncture, cannulation and incision. Circulation 35: 1118, 1967.

3.- Lundsgaard, $C$, and Moller, $E$. Investigations on the oxygen content of cutaneous blood (so called capillary blood). J. Exp. Med. 36: 559, 1922.

4.- Singer, R. B., Shohl, J., Bluemle, D. B. A modification of the Shock and Hasting technique for the simultaneous determination of $\mathrm{pH}, \mathrm{CO}_{2}$ content and cell volume in $0.1 \mathrm{ml}$. aliquots of cutaneous blood. Clin. Chom. 1: 287, 1955.

5.-Siggaard-Andersen, $O$., Engel, $K$. Jorgensen, $K$., and Astris, P. A micromethod for determination of $\mathbf{p H}$, carbon dioxide tension, base excess and standard bicarbonate in capillary blood. Scand. J. Clin. Lab. Invest. 12: 172, 1960.

6.-Astrup, P., Jorgensen, M., Siggaard-Andersen, $O$., and Engel, $K$. The acid-base metabolism. A new approach. Lancet 1: 1035, 1960.
7.-Siggaard-Andersen, O., Jorgensen, K., and Naeraa, $N$. Spectrophotometric determination of oxygen saturation in capillary blood. Scand J. Clin, Lab. Invest. 14: $298,1962$.

8.-Laughlin, D. E, Mc Donald, J. S., and Bedell, G. N. A micro-technique for measurement of $\mathrm{PO}_{2}$ in arterialized earlobe blood. J. Lab. Clin. Med. 64: $330,1964$.

9.-Gambiso, S. R., Goldberg, H. E., and Polangy, $M$. L. A micro method for determination of oxygen saturation by reflexion spectropholometry. Amer. J. Clin. Path. 42: 364, 1964.

10.-Gandy, G., Grann, L., Cunningham, N., Adamsons, $K$, and James, $S$. The validity of $\mathrm{pH}$ and $\mathrm{PCO}_{2}$ measurements in capillary samples in sick and healthy newborn infants. Pediatrics 34: 192, 1964.

11.- Koch, G.: Comparison of $\mathrm{CO}_{2}$ tension, $\mathrm{pH}$ and stindaro bicarbonato in capillary blood and in arterial blood with special respect to relations in patients with impaired cardiovascular and pulmonary function and during exercise. Scand. J. Clin. Lab. Invest. 17: 223, 1965.

12.- Langlands, J. $H$., and Wallace, W, F.: Small blood samples from earlobe puncture. A substitute for arterial puncture. Lancet 11: 315, 1965.

13.- Torjussen, W.: Oxygen saturation in capillary blood.. Scand. J. Clin. Lab. Invest. 17: 505, 1965.

14.-Jung, R. C., Balchun, O. J. and Massey, F. J.: The accuracy venous and capillary blood for the prediction of arterial $\mathrm{pH}, \mathrm{PCO}_{\mathrm{g}}$, and $\mathrm{PO}_{2}$ measurements. Amer. J. Clin. Path 45: 129, 1966.

15.-Koch, G., and Wendel, H.: Comparison of $\mathrm{pH}$, $\mathrm{CO}_{2}$ tension, standard bicarbonate and oxygen tension in capillary blood and in arterial blood during the neonatal period. Acta Paedit. Scand. 56: 10, 1967.

16.-Torjussen, W., and Nitter, H.: $\mathrm{pH}$ and $\mathrm{PCO}_{2}$ in capillary blood from the earlobe. Scand. J. Clin. Lab. Invest. 19: 79, 1967.

17.- Koch, G.: The validity of $\mathrm{PO}_{2}$ measurement in capillary blood as a substitute for arterial $\mathrm{PO}_{2}$. Scand. J. Clin. Lab. Invest. 21: 10, 1968.

18.- Ceruti, E., Contreras, S., Diaz, A., y Núñez, N.: Comparación entre determinaciones de gases $y$ equilibrio ácido-básico en sangre arterial y arterializada en prematuros sanos $y$ con insuficiencia respiratoria. Rey. Chilena Ped, 40: 990, 1969. 
19.-Thomsen, A.: Arterial blood sampling in small infants. Acta Paediat. 53: 237, 1964.

20.-Geubelle, F., and Nicolas-Goldstein, M.: Comparaison entre le $\mathrm{CO}_{2}$ et le $\mathrm{pH}$ de sang. arteriel. Clin. Chim. Acta 3: 480, 1958.

21.-Gambino, S. R.: Collection of capillary blood for simultaneous determinations of arterial $\mathrm{pH}, \mathrm{CO}_{2}$ content, $\mathrm{PCO}_{2}$ and oxygen saturation. Amer. J. Clin. Path. 35: 175, 1961.

22.-Maas, A. H., and Van Heijst, A, N.: A comparison of the $\mathrm{pH}$ of arterial blood with arterialized blood from earlobe with Astrup's micro glasseilectrode. Clin. Chim. Acta 6: 31, 1961 .

23.-Knudsen, E. J, and Hansen, P.: Carhon dioxide tensions in non-arterialized capillary and arterial blood during anesthesia. Acta Anaesth. Scand. 6: $29,1962$.

24.-Howland, W. S., Scweizer, O, and Murphy, T. W.: Estimation of acid-base of venous and arterial blood from capillary samples. Acta Anaesth. Scand. 8: $191,1964$.

25.-Faridy, F. E.: Effect of food and water deprivation on surface activity of lung of rats. J. Appl. Physiol. 29: 493, 1970 .

26.- Mora Parra, J. O., Rojas Garía, J. M., Pardo, F., y Luma-Jaspe, H.: Somatometría en niños de clase socioeconónica baja. Arch. Latinoarner. Nutr. 20: 7. 1970 .
27.-Vallejos, E., y Radrigin, M. E.: Clasificación del estado nutricional. Rev. Chilena Ped. En prensa.

28.- Ardensen, S.: Sampling and storing of blood for determination of acid-base status. Scand, J. Clin. Lab, Invest. 13: 196, 1961.

29.-Rosenthal, T. B.: The effect of temperature of the pH of blood and plasma in vitro. J. Clin. Chem. 173: $25,1948$.

30.-Kundratitz, K.: Die Möglichkeinten der Kliniscben Erkennung drohender ernäbrungestörungen in Saüglingealter und deren Bedeutung für die Toleranzbestianung. Ost. Z. Kinderheil 5; 93, 1950.

31.-Kerpel-Fronius \& Frank. Einige Besonder-heiten der Körperzusammensetzung und Wasserverteilung bei der Säuglingsatrophie. Ann. Paediat. (Basel) 173: 321, 1949.

32.- Utheim, R.: Metabolism studies in infants suffering from chronical nutritional disturbance. Amer. J. Dis. Child. 22: 329, 1921.

33.-Garrow, J. S., Smith, R., and Ward, E. E.: Electrolyts metabolism in severe infantile malnutrition. Pergamon Press. London, 1968.

34.- Ramos Galván, R., Mariscal. C., Viniegra, A, y Pérez-Ortiz, B.: Desnutrición en el niño. Hosp. Infantil Mex. México, D. F., 1969.

35.-Diseases of metabolism, Endocrinology and Nutri. tion. South edition Edited by Philip K. Bondy. W. B. Saunders Company. Philadelphia, 1969. 